\title{
BMJ Open Efficacy and safety of different drug monotherapies for tension-type headache in adults: study protocol for a Bayesian network meta-analysis
}

\author{
Runsheng Xie, ${ }^{1,2}$ Jinhui Tian, ${ }^{3,4}$ Yangyang Wang, ${ }^{1,2}$ Yefeng Cai, ${ }^{5}$ Hui Li ${ }^{1,2}$
}

To cite: Xie R, Tian J, Wang Y, et al. Efficacy and safety of different drug monotherapies for tension-type headache in adults: study protocol for a Bayesian network meta-analysis. BMJ Open 2019;9:e023748. doi:10.1136/ bmjopen-2018-023748

- Prepublication history and additional material for this paper are available online. To view these files, please visit the journal online (http://dx.doi org/10.1136/bmjopen-2018023748).

RX and JT contributed equally.

Received 22 April 2018 Revised 16 0ctober 2018 Accepted 26 November 2018

Check for updates

\section{(c) Author(s) (or their} employer(s)) 2019. Re-use permitted under CC BY-NC. No commercial re-use. See rights and permissions. Published by BMJ.

For numbered affiliations see end of article.

Correspondence to

Dr Hui Li; lihuitcm@126.com

\section{ABSTRACT}

Introduction Tension-type headache (TTH) is the most prevalent neurological disease, with an estimated 1.5 billion cases worldwide. Pharmacotherapy should be considered by patients with TTH who have a limited response to non-pharmacological treatment. However, recommendations for the vast array of therapeutic drugs for TTH partially overlap, with conflicting recommendations for strength in different guidelines; these may confuse the decision-making process of clinicians. Hence, the aims of this study are to analyse the available direct and indirect evidence on different drug monotherapies for TTH in adults, and to generate a treatment ranking according to their efficacy and safety outcomes by using a Bayesian network meta-analysis (NMA).

Methods and analysis We will systematically search the Cochrane Library, PubMed, Web of Science, Embase, China Biomedical Literature Database, International Clinical Trials Registry Platform and other resources for eligible studies. Randomised controlled trials on different drug monotherapies for TTH will be included. Two review authors ( $R X$ and $\mathrm{YW}$ ) will independently search and select the studies, extract the data and assess the risk of bias. A Bayesian NMA will afterwards be conducted to pool the effect measures across all types of monotherapy drugs. The ranking probabilities of the efficacy and safety of different drug monotherapies will be estimated. Heterogeneity will be quantified using the $Q$ statistic and the $\mathrm{I}^{2}$ index. Inconsistency between direct and indirect evidence will be assessed by the node-splitting model. In addition, the overall quality of evidence will be assessed using the Grading of Recommendations Assessment, Development and Evaluation approach.

Ethics and dissemination No ethical issues are foreseen. The results will be published in a peer-reviewed journal, which will be disseminated electronically and in print. PROSPERO registration number CRD42018090554.

\section{INTRODUCTION}

Over the past 25 years, the burden of neurological disease has increased constantly, and neurological diseases have become a major cause of disability and death worldwide. ${ }^{1}$ Tension-type headache (TTH) is the most prevalent neurological disease, with an

\section{Strengths and limitations of this study}

- This Bayesian network meta-analysis will provide a comprehensive summary of the direct and indirect evidence on the efficacy and safety of different drug monotherapies for tension-type headache (TTH) in adults.

- The relative ranking results of the efficacy and safety outcomes will facilitate patients, clinicians and healthcare providers in the selection of the available drug monotherapies by providing the highest level of evidence.

- This protocol is drafted in accordance with the Preferred Reporting Items for Systematic Review and Meta-Analysis Protocols 2015 statement and has been registered with the International prospective register of systematic reviews (PROSPERO).

- The overall quality of evidence will be assessed using the Grading of Recommendations Assessment, Development and Evaluation approach.

- This research will exclude non-English, non-Chinese and non-randomised controlled trials, and the publications investigating combination therapies for $\mathrm{TTH}$ will also be limited.

estimated 1.5 billion cases globally. ${ }^{12}$ TTH is generally a diffuse, mild-to-moderate pain in the head, often described as feeling like a tight band around the head. TTH may be associated with considerable disability, low effectiveness at work, absenteeism or decreased learning ability, and may have a great impact on the patient's quality of life. ${ }^{2}$ Pharmacotherapy should be considered or added for patients with TTH who show a limited response to non-pharmacological treatment. $^{3}$

Since 1995, TTHs have been divided into episodic TTH (ETTH) and chronic TTH (CTTH) subtypes; these were introduced in the first edition of the International Classification of Headache Disorders of the International Headache Society (IHS). ${ }^{4}$ Our preliminary search found that the 
recommendations for therapeutic drugs for patients with ETTH or CTTH varied widely between different guidelines, partially overlapped and exhibited variation in recommended strength (online supplementary appendix 1) ${ }^{5-15}$ That is, either the same drug was recommended at different strength in different guidelines, or different guidelines recommended different pharmaceuticals. For example, ibuprofen and ketoprofen were considered to be level A in the European Federation of Neurological Societies guidelines, although the Italian guidelines suggested these two analgesics were at the level II recommendation. ${ }^{814}$

Thus far, evidence for the acute treatment of ETTH and the prophylactic treatment of CTTH of direct headto-head comparison among all treatments is scarce. In addition, conventional pairwise meta-analyses as a means of summarising evidence do not allow for the inclusion of data that have not been direct comparisons. Hopefully, previous studies have shown that the combined results of direct evidence and indirect evidence can improve accuracy for treatments that have been directly evaluated. ${ }^{16}$ Therefore, to assess the relationships between all treatments, a network meta-analysis (NMA) will be necessary for the integration of direct and indirect evidence from multiple treatment comparisons. ${ }^{17}$

The relative efficacy and safety among different types of drugs and between different drugs of the same type for the treatment of ETTH and CTTH are not yet clear. Therefore, clinicians may be confused when making decisions on pharmaceuticals. Hence, the aims of this study are to synthesise the available direct and indirect evidence on the different drug monotherapies for ETTH and CTTH in adults, and to generate a treatment ranking based on their efficacy and safety outcomes by using an NMA.

\section{METHODS AND ANALYSIS}

This protocol is drafted in accordance with the Preferred Reporting Items for Systematic Review and Meta-Analysis Protocols 2015 statement. ${ }^{18}$ It has been registered with the International prospective register of systematic reviews (PROSPERO).

\section{Criteria for included studies}

Participants and settings

The participants studied by this review must be adult patients ( $\geq 18$ years of age) with TTH (either ETTH or CTTH).

The diagnosis criteria for TTH should be developed by professional organisations or agencies (eg, the IHS $)^{19}$; they can clearly classify TTH into ETTH and CTTH and reasonably distinguish TTH from other types of headache.

Only data from participants with ETTH or CTTH will be analysed. Studies and trials including participants with 'mixed' or 'combination' TTH and other types of headache will be excluded. There will be no limitations on participants' gender, race and nationality.
Interventions

In our preliminary studies, we searched the relevant databases, electronic databases and websites for guidelines containing ETTH or CTTH drug monotherapies. These monotherapies were extracted to the 'ETTH and CTTH drug monotherapies list' (table 1).

Each intervention from the included studies shall match at least one monotherapy of the 'ETTH and CTTH drug monotherapies table'. There will be no restriction on dose.

Studies solely investigating on non-pharmacological interventions, or on combinations of drugs instead of monotherapies, will be excluded.

\section{Comparators}

The comparator(s)/control of the included studies shall involve at least one monotherapy from the "ETTH and CTTH drug monotherapies list' or blank/placebo control.

\section{Outcome measures \\ Primary outcome}

The primary efficacy outcomes will be pain-free at 2 hours, sustained freedom from pain at 24 hours and Visual Analogue Scale score. The primary safety outcomes will be the incidence of adverse events, gastrointestinal adverse reactions and addiction to drugs.

\section{Secondary outcomes}

The possible secondary efficacy outcomes are as follows: (1) changes in patient-reported headache frequency, duration and intensity; and (2) functional health status and health-related quality of life (eg, 36-Item Short Form Survey). The possible secondary safety outcomes are (1) liver-kidney function indicators and (2) faecal occult blood.

\section{Study design and publication types}

Randomised controlled trial (RCT) studies in any setting using different drug monotherapies for ETTH or CTTH in adults will be included. We will exclude publications that were not peer-reviewed, such as letters, comments and conference proceedings.

\section{Information sources and search strategy}

We will develop search strategies for each electronic database, based on the search strategy developed for PubMed (online supplementary appendix 2), with appropriate revisions for each database. The following databases will be searched: Cochrane Library, PubMed, Web of Science, Embase, China Biomedical Literature Database and International Clinical Trials Registry Platform. We will also search other resources for eligible studies. The search dates will be from the establishment of the respective library to 15 March 2018. The languages will be limited to English and Chinese. In addition, we will also hand-search the reference lists of all eligible articles for additional studies if they meet our eligibility criteria. 


\begin{tabular}{|c|c|c|}
\hline $\begin{array}{l}\text { Subtype } \\
\text { of TTH }\end{array}$ & $\begin{array}{l}\text { Drug } \\
\text { classification }\end{array}$ & Drug treatment \\
\hline \multirow[t]{15}{*}{ ETTH } & \multirow[t]{9}{*}{$\begin{array}{l}\text { Non-steroidal anti- } \\
\text { inflammatory drugs }\end{array}$} & $\begin{array}{l}\text { Aspirin/acetylsalicylic } \\
\text { acid }\end{array}$ \\
\hline & & Acetaminophen/paracetamol \\
\hline & & Lumiracoxib \\
\hline & & Ibuprofen \\
\hline & & Ketoprofen \\
\hline & & Naproxen \\
\hline & & Diclofenac \\
\hline & & Diclofenac-K \\
\hline & & Metamizole/Dipyrone \\
\hline & \multirow[t]{3}{*}{ Analgesics } & Codeine \\
\hline & & Dihydrocodeine \\
\hline & & Dextropropoxyphene \\
\hline & \multirow[t]{2}{*}{ Antiemetics } & Metoclopramide \\
\hline & & Chlorpromazine \\
\hline & Supplementary & Tiger balm \\
\hline \multirow[t]{27}{*}{ CTTH } & \multirow[t]{17}{*}{ Antidepressants } & Amitriptyline \\
\hline & & L-5-Hydroxytryptophan \\
\hline & & Fluvoxamine \\
\hline & & Venlafaxine \\
\hline & & Clomipramine \\
\hline & & Mirtazapine \\
\hline & & Maprotiline \\
\hline & & Mianserin \\
\hline & & Desipramine \\
\hline & & Fluoxetine \\
\hline & & Paroxetine \\
\hline & & Nefazodone \\
\hline & & Ritanserin \\
\hline & & Sulpiride \\
\hline & & Dothiepin/Prothiaden \\
\hline & & Nortriptyline \\
\hline & & Protriptyline \\
\hline & \multirow[t]{4}{*}{ Antiepileptics } & Sodium valproate \\
\hline & & Topiramate \\
\hline & & Gabapentin \\
\hline & & Levetiracetam \\
\hline & \multirow[t]{3}{*}{ Anxiolytics } & Diazepam/Valium \\
\hline & & Alprazolam \\
\hline & & Buspirone \\
\hline & \multirow[t]{3}{*}{ Narcotics } & Tizanidine \\
\hline & & Cyclobenzaprine \\
\hline & & $\begin{array}{l}\text { Botulinum toxin A/ } \\
\text { OnabotulinumtoxinA }\end{array}$ \\
\hline
\end{tabular}

CTTH, chronic TTH; ETTH, episodic TTH; TTH, tension-type headache.

\section{Study selection}

Two review authors (RX and YW) will independently screen the titles/abstracts of all studies retrieved according to the search strategy and those obtained from additional sources to identify the studies suitable for the inclusion criteria mentioned above. Afterwards, the full text of the remaining studies will also be retrieved and independently assessed for eligibility. Any disagreement between them will be resolved by discussion or by referral to a third reviewer for a final decision.

\section{Data extraction}

We will design a prepiloted data extraction form to extract data from the included studies for study quality assessment and evidence synthesis. Using this form, two authors (RX and YW) will independently extract data from each study. Any disagreement that occurred will be resolved by mutual discussion or by referral to a third reviewer for a final decision. The extracted information will include basic information on the study; characteristics of the study; details of the intervention and control group; outcomes measures and their data; risk of bias (quality) assessment information; and other relevant information.

\section{Risk of bias assessment}

Two review authors (RX and YW) will independently assess the risk of bias in included studies, using the criteria outlined in the Cochrane Handbook for Systematic Reviews of Interventions. ${ }^{16}$

Each study will be assessed on the following aspects:

1. Random sequence generation (to assess the domain of selection bias). We will assess the method used to generate the allocation sequence in sufficient detail as low risk of bias (the investigators describe a random component in the sequence generation process); high risk of bias (the investigators describe a non-random component in the sequence generation process); or unclear risk of bias (insufficient information about the sequence generation process to permit judgement).

2. Allocation concealment (to assess the domain of selection bias). We will assess the method used to conceal the allocation sequence in sufficient detail as low risk of bias (participants and investigators enrolling participants could not foresee assignment); high risk of bias (participants or investigators enrolling participants could possibly foresee assignments and thus introduce selection bias); or unclear risk of bias (insufficient information to permit judgement).

3. Blinding of participants and personnel (to assess the domain of performance bias). We will assess the method used to blind study participants and personnel from knowledge of which intervention a participant received as low risk of bias (the outcome is not likely to be influenced by lack of blinding, or the blinding could not have been broken); high risk of bias (the outcome is likely to be influenced by lack of blinding, or the blinding could have been broken); or unclear 
risk of bias (insufficient information to permit judgement, or the study did not address this outcome).

4. Blinding of outcome assessment (to assess the domain of detection bias). We will assess the method used to blind outcome assessors from the knowledge of which intervention a participant received as low risk of bias (the outcome measurement is not likely to be influenced by lack of blinding, or the blinding could not have been broken); high risk of bias (the outcome measurement is likely to be influenced by lack of blinding, or the blinding could have been broken); or unclear risk of bias (insufficient information to permit judgement, or the study did not address this outcome).

5. Incomplete outcome data (to assess the domain of attrition bias). We will assess the completeness of outcome data for each main outcome as low risk of bias (no missing outcome data, or missing outcome data unlikely to have a clinically relevant impact on observed effect size); high risk of bias (missing outcome data likely to be related to the true outcome, or missing outcome data sufficient to induce clinically relevant bias in observed effect size); or unclear risk of bias (insufficient reporting of attrition/exclusion to permit judgement, or the study did not address this outcome).

6 . Selective reporting (to assess the domain of reporting bias). We will assess the possibility of selective outcome reporting by the review authors as low risk of bias (the study protocol is available and all of the study outcomes are prespecified, or the study protocol is not available but it is clear that the published reports include all expected outcomes); high risk of bias (not all of the prespecified primary outcomes of the study have been reported, or one or more primary outcomes are reported using measurements, analysis methods or subsets of the data that were not prespecified); or unclear risk of bias (insufficient information to permit judgement).

7. Other sources of bias (to assess the domain of other bias). We will assess any important concerns about bias not addressed in the other domains in the tool as low risk of bias (the study appears to be free of other sources of bias); high risk of bias (there is at least one important risk of bias); or unclear risk of bias (insufficient information to assess whether an important risk of bias exists, or insufficient rationale or evidence that an identified problem will introduce bias).

\section{Statistical analysis}

We will descriptively summarise the included studies based on the study characteristics, patient characteristics, intervention and outcome measures, and our assessment of the risk of bias. If quantitative synthesis is not appropriate, we will describe the results of the systematic review.

We will calculate the risk ratio and its $95 \%$ CIs for dichotomous data, and the mean differences with $95 \%$ CIs for continuous data. Weighted mean differences will be used for data measured on the same scale with the same units; otherwise, standardised mean differences will be used. When lacking head-to head comparisons, indirect treatment comparison meta-analysis will be retrieved from the available evidence.

We will perform the NMA in the Bayesian framework using the Markov chain Monte Carlo method. In our NMA of TTH treatment efficacy and safety, effect measures across all types of drug monotherapies will be pooled. Convergence of the simulations will be evaluated using trace plots, density plots and Brooks-GelmanRubin diagnosis plots. ${ }^{20}$ In this study, both fixed-effects and random-effects models in the Bayesian NMA will be considered based on the results of the deviance information criterion. Moreover, the ranking probability of the efficacy and safety of different drug monotherapies will be estimated for the acute treatment of ETTH and the prophylactic treatment of CTTH. The results of rankograms, ranking probabilities plots and evidence network plots will be displayed graphically. Cumulative ranking will be estimated by the surface under the cumulative ranking curve (SUCRA) for each TTH treatment. SUCRA will be 1 when a treatment is certain to be the best and 0 when a treatment is certain to be the worst, with higher values indicating better efficacy or safety. ${ }^{21}$

\section{Assessment of heterogeneity}

Heterogeneity will be quantified with $Q$ statistic and $I^{2}$ index. We will consider $\mathrm{p}<0.1$ or $\mathrm{I}^{2} \geq 50 \%$ indicative of at least moderate heterogeneity. ${ }^{22}$ Under this circumstance, the random-effect model will be used. Otherwise, the fixed-effect model will be used.

\section{Assessment of inconsistency}

Inconsistency between direct and indirect evidence will be assessed by the node-splitting model, which is a straightforward interpretation, contrasting estimates from both direct and indirect evidence. ${ }^{23}$ Values of $\mathrm{p}<0.05$ indicate inconsistency between direct and indirect estimates in a specific closed loop.

\section{Assessment of similarity}

All indirect analyses are based on the underlying assumption that the study populations in the trials being compared are sufficiently similar to be pooled, akin to meta-analyses. ${ }^{24}$ The similarities in the clinical and methodological characteristics, such as baseline data for patients and trial design, between studies will be qualitatively compared.

\section{Sensitivity analysis}

We will assess the robustness of our results through a series of sensitivity analyses: the exclusion trials with a high risk of bias, the iterative removal of one study at a time, and the use of both fixed and random-effects models.

Assessment of publication bias and small-study effects

We will use funnel plots for each treatment comparison separately to assess for publication bias if there are 10 or more studies reporting on a particular outcome. Small-study effects will be tested within a network 
meta-regression model that distinguishes studies based on their size.

\section{Subgroup analysis}

Possible subgroup analyses will be performed based on the age of patients and the route of drug administration.

\section{Software}

The NMA in the Bayesian framework will be conducted using JAGS V.4.2.0, with 'gemtc', 'R2WinBUGS', 'lattice' and 'coda' packages in R V.3.4.4. ${ }^{25}$

\section{Assessment of quality of evidence}

The overall quality of evidence will be assessed using the Grading of Recommendations Assessment, Development and Evaluation approach on the efficacy and safety of different drug monotherapies for TTH in adults. The quality of RCT evidence will be classified into high, moderate, low or very low quality evidence, depending on the presence of these five factors: (1) limitations in the design and implementation; (2) indirectness of evidence; (3) unexplained heterogeneity or inconsistency of results; (4) imprecision of results; and (5) high probability of publication bias. ${ }^{16}$

\section{Patient and public involvement}

There was no patient or public involvement in the development of this manuscript. Following completion of this work, we will disseminate our findings through open-access publications.

\section{DISCUSSION}

Among the different types of headaches, TTH is probably the most prevalent, but the least studied. ${ }^{326}$ According to the preliminary guideline search results, at minimum of 11 guidelines currently recommend more than 40 different drug monotherapies for the acute treatment of ETTH and the prophylactic treatment of CTTH. However, these recommendations cannot provide a clear answer on the best choice for the initial treatment of ETTH and CTTH owing to a lack of consistency. Therefore, we have proposed an NMA to quantitatively synthesise the available direct and indirect evidence on the different drug monotherapies for ETTH and CTTH. The relative ranking of efficacy and safety outcomes of each competing treatment will be presented. We expect that the results of this research will facilitate the decision making by patients, clinicians and healthcare providers in the treatment of patients with TTH with pharmaceuticals.

The limitations of this research will be noted. First, the exclusion of non-English and non-Chinese studies may cause publication bias. Second, we will exclude non-RCT publications to support our intention to include only higher quality evidence. Finally, this study did not include publications of combination therapy for TTH, which may affect the generalisability of this study.

\section{Author affiliations}

${ }^{1}$ Department of Standardization of Chinese Medicine, The Second Affiliated Hospital of Guangzhou University of Chinese Medicine (Guangdong Provincial Hospital of Chinese Medicine; Guangdong Provincial Academy of Chinese Medical Sciences), Guangzhou, China

${ }^{2}$ Engineering and Technology Research Center of Standardization of Traditional Chinese Medicine, Guangzhou, China

${ }^{3}$ Evidence-Based Medicine Center, Lanzhou University, Lanzhou, China

${ }^{4}$ Key Laboratory of Evidence-Based Medicine and Knowledge Translation of Gansu Province, Lanzhou, China

${ }^{5}$ Department of Internal Neurology, The Second Affiliated Hospital of Guangzhou University of Chinese Medicine (Guangdong Provincial Hospital of Chinese Medicine; Guangdong Provincial Academy of Chinese Medical Sciences), Guangzhou, China

Acknowledgements We would like to thank Editage (www.editage.com) for its linguistic assistance during the preparation of this manuscript. We would also like to acknowledge Dr Yu-Qing Zhang and Mr Jake Chen for their useful suggestions.

Contributors RX, JT and HL conceived the study and drafted the manuscript. JT and YW provided search strategies and professional advice. RX and YW implemented a preliminary search. JT and HL provided guidance on the NMA methodology. YC and HL provided expertise on treatments, outcomes and related knowledge on TTH. All authors read, critically reviewed and approved the final manuscript as submitted.

Funding This work was supported by Special Research Fund for Traditional Chinese Medicine Science and Technology of the Guangdong Provincial Hospital of Chinese Medicine (grant number YN2015MS22).

Competing interests None declared.

Patient consent for publication Not required.

Provenance and peer review Not commissioned; externally peer reviewed.

Open access This is an open access article distributed in accordance with the Creative Commons Attribution Non Commercial (CC BY-NC 4.0) license, which permits others to distribute, remix, adapt, build upon this work non-commercially, and license their derivative works on different terms, provided the original work is properly cited, appropriate credit is given, any changes made indicated, and the use is non-commercial. See: http://creativecommons.org/licenses/by-nc/4.0/.

\section{REFERENCES}

1. Feigin VL, Abajobir AA, Abate KH, et al. Global, regional, and national burden of neurological disorders during 1990-2015: a systematic analysis for the Global Burden of Disease Study 2015. Lancet Neurol 2017; $16: 877-97$.

2. Crystal SC, Robbins MS. Epidemiology of tension-type headache. Curr Pain Headache Rep 2010;14:449-54.

3. Jensen $\mathrm{RH}$. Tension-type headache - the normal and most prevalent headache. Headache 2018;58:339-45.

4. Schoenen J. Guidelines for trials of drug treatments in tension-type headache. First edition: International Headache Society Committee on Clinical Trials. Cephalalgia 1995;15:165-79.

5. Simpson DM, Hallett M, Ashman EJ, et al. Practice guideline update summary: Botulinum neurotoxin for the treatment of blepharospasm, cervical dystonia, adult spasticity, and headache: Report of the Guideline Development Subcommittee of the American Academy of Neurology. Neurology 2016;86:1818-26.

6. British Association for the Study of Headache (BASH). Guidelines for all healthcare professionals in the diagnosis and management of migraine, tension-type, cluster and medication-overuse headache. 3rd edn, 2007.

7. Vuković Cvetković V, Kes VB, Serić V, et al. Report of the Croatian Society for Neurovascular Disorders, Croatian Medical Association. Evidence based guidelines for treatment of primary headaches--2012 update. Acta Clin Croat 2012;51:323-78.

8. Bendtsen L, Evers S, Linde M, et al. EFNS guideline on the treatment of tension-type headache - report of an EFNS task force. Eur $J$ Neurol 2010;17:1318-25.

9. Steiner TJ, Paemeleire K, Jensen R, et al. European principles of management of common headache disorders in primary care. $J$ Headache Pain 2007;8(Suppl 1):S3-47.

10. Moisset X, Mawet J, Guegan-Massardier E, et al. French Guidelines For the Emergency Management of Headaches. Rev Neurol 2016;172:350-60. 
11. Institute for Clinical Systems Improvement (ICSI). Health care guideline: diagnosis and treatment of headache. 10th edn, 2011.

12. National Institute for Health and Care Excellence. Diagnosis and management of headache in young people and adults. CG150. London: NICE, 2012.

13. Scottish Intercollegiate Guidelines Network (SIGN). The diagnosis and management of headache in adults. Guideline No 107.) Edinburgh: SIGN 2008.

14. Sarchielli P, Granella F, Prudenzano MP, et al. Italian guidelines for primary headaches: 2012 revised version. $J$ Headache Pain 2012;13:31-70.

15. Becker WJ, Findlay T, Moga C, et al. Guideline for primary care management of headache in adults. Can Fam Physician 2015;61:670-9.

16. Higgins JPT, Green S. Cochrane handbook for systematic reviews of interventions version 5.1.0: The Cochrane Collaboration, 2011.

17. Mavridis D, Giannatsi M, Cipriani A, et al. A primer on network metaanalysis with emphasis on mental health. Evid Based Ment Health 2015;18:40-6.

18. Shamseer L, Moher D, Clarke M, et al. Preferred reporting items for systematic review and meta-analysis protocols (PRISMA-P) 2015: elaboration and explanation. BMJ 2015;349:g7647.
19. Headache Classification Committee of the International Headache Society (IHS). The international classification of headache disorders, 3rd edition (beta version). Cephalalgia 2013;33:629-808.

20. Brooks SP, Gelman A. Alternative methods for monitoring convergence of iterative simulations. J Comput Graph Stat 1998;7:434-45.

21. Salanti G, Ades AE, loannidis JP. Graphical methods and numerical summaries for presenting results from multiple-treatment metaanalysis: an overview and tutorial. J Clin Epidemiol 2011;64:163-71.

22. Higgins JP, Thompson SG, Deeks JJ, et al. Measuring inconsistency in meta-analyses. BMJ 2003;327:557-60.

23. van Valkenhoef $\mathrm{G}$, Dias $\mathrm{S}$, Ades $\mathrm{AE}$, et al. Automated generation of node-splitting models for assessment of inconsistency in network meta-analysis. Res Synth Methods 2016;7:80-93.

24. Kim H, Gurrin L, Ademi Z, et al. Overview of methods for comparing the efficacies of drugs in the absence of head-to-head clinical trial data. Br J Clin Pharmacol 2014;77:116-21.

25. R Core Team. R: A language and environment for statistical computing. Vienna, Austria: R Foundation for Statistical Computing, 2018.

26. Mesa-Jiménez JA, Lozano-López C, Angulo-Díaz-Parreño S, et al. Multimodal manual therapy vs. pharmacological care for management of tension type headache: A meta-analysis of randomized trials. Cephalalgia 2015;35:1323-32. 